\title{
Linear-in-frequency optical conductivity in GdPtBi due to transitions near the triple points
}

\author{
F. Hütt, ${ }^{1}$ A. Yaresko, ${ }^{2}$ M. B. Schilling, ${ }^{1}$ C. Shekhar, ${ }^{3}$ C. Felser,${ }^{3}$ M. Dressel,${ }^{1}$ and A. V. Pronin ${ }^{1}$ \\ ${ }^{1}$ 1. Physikalisches Institut, Universität Stuttgart, 70569 Stuttgart, Germany \\ ${ }^{2}$ Max-Planck-Institut für Festkörperforschung, 70569 Stuttgart, Germany \\ ${ }^{3}$ Max-Planck-Institut für Chemische Physik fester Stoffe, 01187 Dresden, Germany
}

(Dated: October 23, 2018)

\begin{abstract}
The complex optical conductivity of the half-Heusler compound GdPtBi is measured in a frequency range from 20 to $22000 \mathrm{~cm}^{-1}(2.5 \mathrm{meV}-2.73 \mathrm{eV})$ at temperatures down to $10 \mathrm{~K}$ in zero magnetic field. We find the real part of the conductivity, $\sigma_{1}(\omega)$, to be almost perfectly linear in frequency over a broad range from 50 to $800 \mathrm{~cm}^{-1}(\sim 6-100 \mathrm{meV})$ for $T \leq 50 \mathrm{~K}$. This linearity strongly suggests the presence of three-dimensional linear electronic bands with band crossings (nodes) near the chemical potential. Band-structure calculations show the presence of triple points, where one doubly degenerate and one nondegenerate band cross each other in close vicinity of the chemical potential. From a comparison of our data with the optical conductivity computed from the band structure, we conclude that the observed nearly linear $\sigma_{1}(\omega)$ originates as a cumulative effect from all the transitions near the triple points.
\end{abstract}

Heusler materials are currently well recognized for their wide range of spectacular electronic and magnetic properties [1]. The high tunability of these compounds allows designing materials with properties on demand for future functioning applications [2, 3]. Recently, band inversion and topologically nontrivial electronic states have been intensively studied in (half-)Heusler compounds with strong spin-orbit coupling (SOC) [4-11].

Among other half-Heusler compounds with strong SOC, GdPtBi occupies a special place: the hallmarks of a Weyl-semimetal (WSM) state, such as negative magnetoresistance and the planar Hall effect, are vividly developed in this material and are assigned to manifestations of the chiral anomaly [12 14]. It has been proposed that the band structure of GdPtBi in zero magnetic field can be sketched as two degenerate parabolic bands touching each other at the $\Gamma$ point of the Brillouin zone (BZ) [12]. A moderate external magnetic field splits the bands. This leads to linear-band crossings and a WSM state, which enables negative longitudinal magnetoresistance 12, 13. and the planar Hall effect [14]. Most recent densityfunctional-theory calculations, though, forecast linearband crossings even in zero magnetic field 15]. These nodes are, however, different from the Dirac and Weyl points: in the GdPtBi case, one doubly and one nondegenerate band cross each other, forming the so-called triple points [16]. Angular-resolved photoemission reveals linear electronic bands in GdPtBi, but these bands are mostly assigned to the surface states [8].

Because of large penetration depths, optical methods are more sensitive to bulk properties [17]. It is also known that optical transitions between bands with linear dispersion relations manifest themselves as characteristic features in the optical response [18 23]. For example, crossing three-dimensional (3D) linear bands are supposed to show up as linear-in-frequency conductivity, $\sigma_{1}(\omega) \propto \omega$. Such linearity of $\sigma_{1}(\omega)$ - unusual for conventional metals and semiconductors - is currently widely considered as a hallmark for solid-state 3D Dirac physics and has indeed been observed in a number of nodal semimetals 24 28]. Therefore, it is tempting to probe the optical response of GdPtBi and to compare it with theory predictions.

In this paper, we report on measurements of the optical conductivity in GdPtBi. We find $\sigma_{1}(\omega)$ to be linear in a broad frequency range: at $T \leq 50 \mathrm{~K}$, the linearity spans from $\sim 100 \mathrm{meV}$ down to a few $\mathrm{meV}$. We calculate $\sigma_{1}(\omega)$ from the GdPtBi band structure and, by comparing the experimental and the computed conductivity, demonstrate that the linear-in-frequency $\sigma_{1}(\omega)$ is due to electronic transitions between the bands in the vicinity of the triple points.

GdPtBi single crystals were grown by the solution method from a Bi flux. Freshly polished pieces of Gd, $\mathrm{Pt}$, and $\mathrm{Bi}$, each of purity larger than $99.99 \%$, in the ratio $\mathrm{Gd}: \mathrm{Pt}: \mathrm{Bi}=1: 1: 9$ were placed in a tantalum crucible and sealed in a dry quartz ampoule under 3 mbar partial pressure of argon. The filled ampoule was heated at a rate of $100 \mathrm{~K} / \mathrm{hr}$ up to $1200^{\circ} \mathrm{C}$, followed by 12 hours of soaking at this temperature. For crystal growth, the temperature was slowly reduced by $2 \mathrm{~K} / \mathrm{hr}$ to $600^{\circ} \mathrm{C}$. Extra Bi flux was removed by decanting it from the ampoule at $600^{\circ} \mathrm{C}$. Overall, the crystal-growth procedure followed closely the ones described in Refs. [11, 29]. The crystals' composition and structure (noncentrosymmetric $F \overline{4} 3 m$ space group) were checked by energy dispersive $\mathrm{x}-$ ray analysis and Laue diffraction, respectively.

Our optical reflectivity measurements were conducted on a single crystal of lateral dimensions of $\sim 2 \times 1.1 \mathrm{~mm}^{2}$ with a shiny (111) surface (Fig. 1); the sample thickness was around $0.8 \mathrm{~mm}$. Standard four-point dc-resistivity and Hall measurements, performed on a smaller piece (a Hall bar) cut from the specimen used for optics, indicated a semiconducting behavior with a well-known antiferromagnetic transition at $9 \mathrm{~K}[29]$. Hall measurements 
show $p$-type conduction and a very low carrier density of $6 \times 10^{-17} \mathrm{~cm}^{-3}$ at $T \rightarrow 0$ (cf. different samples from Ref. [12]). All optical experiments reported here are made in the paramagnetic state $(T \geq 10 \mathrm{~K})$, where the Dirac physics of GdPtBi is primarily discussed 12 15].

Optical reflectivity $R(\nu)$ was measured at 10 to $300 \mathrm{~K}$ over a frequency range from $\nu=\omega /(2 \pi c)=20$ to 22000 $\mathrm{cm}^{-1}(2.5 \mathrm{meV}-2.73 \mathrm{eV})$ using two Fourier-transform infrared spectrometers. The spectra in the far-infrared $\left(20-700 \mathrm{~cm}^{-1}\right)$ were recorded by a Bruker IFS $113 \mathrm{v}$ spectrometer. Here, an in-situ gold evaporation technique was utilized for reference measurements [30]. At frequencies above $700 \mathrm{~cm}^{-1}$, a Bruker Hyperion microscope attached to a Bruker Vertex 80v spectrometer was used. Freshly evaporated gold mirrors served as references in this setup. Complex optical conductivity was obtained from $R(\nu)$ using Kramers-Kronig transformations [17]. High-frequency extrapolations were made utilizing the x-ray atomic scattering functions 31]. At low frequencies, we used the same procedure as recently applied for materials with highly mobile carriers [32, 33]: we fitted the $R(\nu)$ spectra with a set of Lorentzians 34] and then used the results of these fits between $\nu=0$ and $20 \mathrm{~cm}^{-1}$ as zero-frequency extrapolations for subsequent Kramers-Kronig transformations.

Figure2 displays an overview of the results obtained in our optical investigations. Panel (a) shows the reflectivity for all measurement temperatures. Panels (b) and (c) represent (the real parts of) the dielectric constant $\varepsilon_{1}(\nu)$ and the optical conductivity $\sigma_{1}(\nu)$, respectively. Finally, panel (d) demonstrates the skin depth $\delta(\nu)$ of our sample at 10 and $300 \mathrm{~K}$ (curves for intermediate temperatures

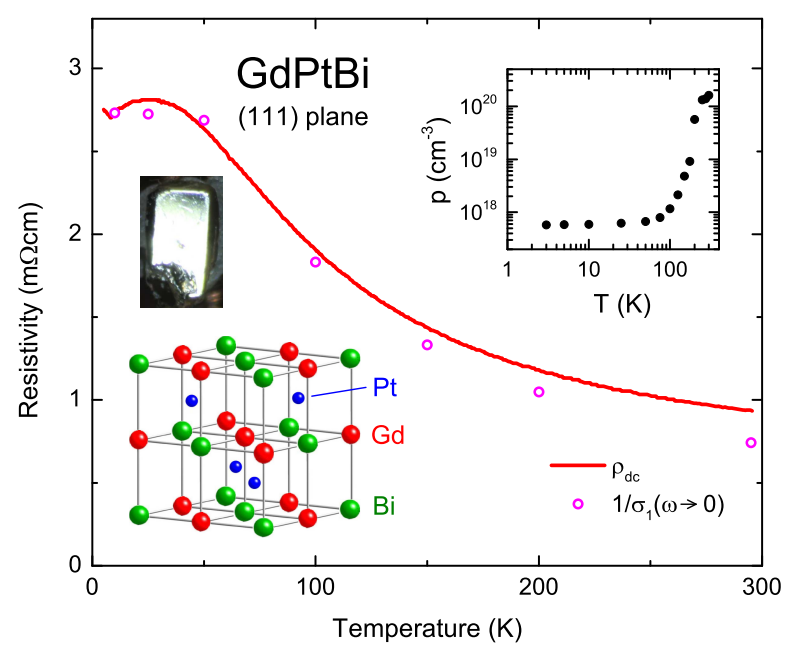

FIG. 1. Temperature-dependent dc resistivity (solid line) of $\mathrm{GdPtBi}$ and the inverse values of its optical conductivity at $\omega \rightarrow 0$ (open dots). Inset: carrier concentration $p$ obtained from Hall measurements. The sample used in this work is also shown alongside the GdPtBi structure.
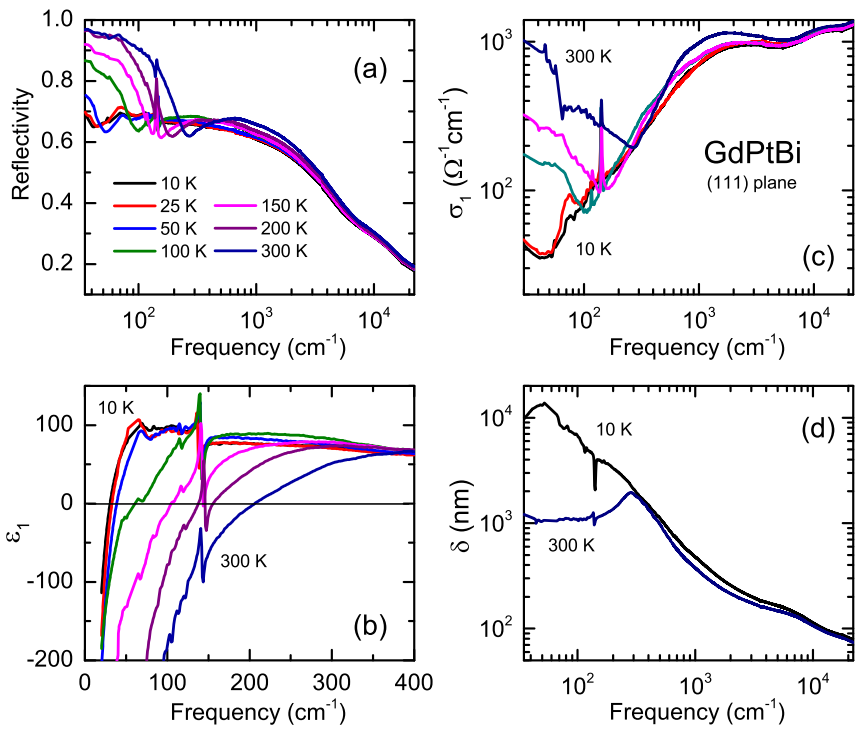

FIG. 2. Reflectivity (a), dielectric constant $\varepsilon_{1}$ (b), optical conductivity $\sigma_{1}(\mathrm{c})$, and skin depth $\delta(\mathrm{d})$ of GdPtBi at different temperatures as indicated. The low-frequency portion of $\sigma_{1}(\nu)$ is magnified in Fig. 3

lie in between these ones). Important is that the skin depth exceeds $50 \mathrm{~nm}$ for all measured temperatures and frequencies. In the most interesting low-energy region, it is in the micrometer range. Hence, our optical measurements reflect bulk properties.

A sharp phonon peak is seen in all data sets at $\sim 140$ $\mathrm{cm}^{-1}$. Another phonon at $\sim 115 \mathrm{~cm}^{-1}$ is weak, but resolvable, especially in $\varepsilon_{1}(\nu)$ [panel (b)]. The frequency positions of both phonon modes have only marginal temperature dependence. No other phonons are detected, in agreement with group analysis, which predicts two infrared-active optical modes for the half-Heusler structure [35]. All other features of the optical response are due to intra- or interband electronic transitions, as discussed below.

A temperature-dependent plasma edge dominates the low-energy part $\left(\nu<300 \mathrm{~cm}^{-1}\right)$ of the reflectivity spectra [panel (a)]. The edge corresponds to the screened plasma frequency of free carriers, $\nu_{\mathrm{pl}}^{\mathrm{scr}}$ [17], and is also seen in panel (b) as zero crossings of the $\varepsilon_{1}(\nu)$ curves. From the same panel, it can also be noted that the background dielectric constant $\varepsilon_{\infty}$ is rather high, around 70 - 100. This leads to a low unscreened plasma frequency $\nu_{\mathrm{pl}}=\nu_{\mathrm{pl}}^{\mathrm{scr}} \sqrt{\varepsilon_{\infty}}$ (for example, $\nu_{\mathrm{pl}} \approx 300 \mathrm{~cm}^{-1}$ at $10 \mathrm{~K}$ ), in agreement with the low free-carrier concentration found in Hall measurements. The free-electron contribution to the optical conductivity [panel (c)] is seen as a Drude-like mode at the lowest frequencies. At lower temperatures, this mode narrows and loses its spectral weight in accordance with decreasing $\nu_{\mathrm{pl}}^{\mathrm{scr}}$ at $T \rightarrow 0$. As $T \rightarrow 0 \mathrm{~K}$, only marginal traces of the free-carrier (intraband) contribution are seen in the recorded $\sigma_{1}(\nu)$ spectra: above $\sim 50$ 

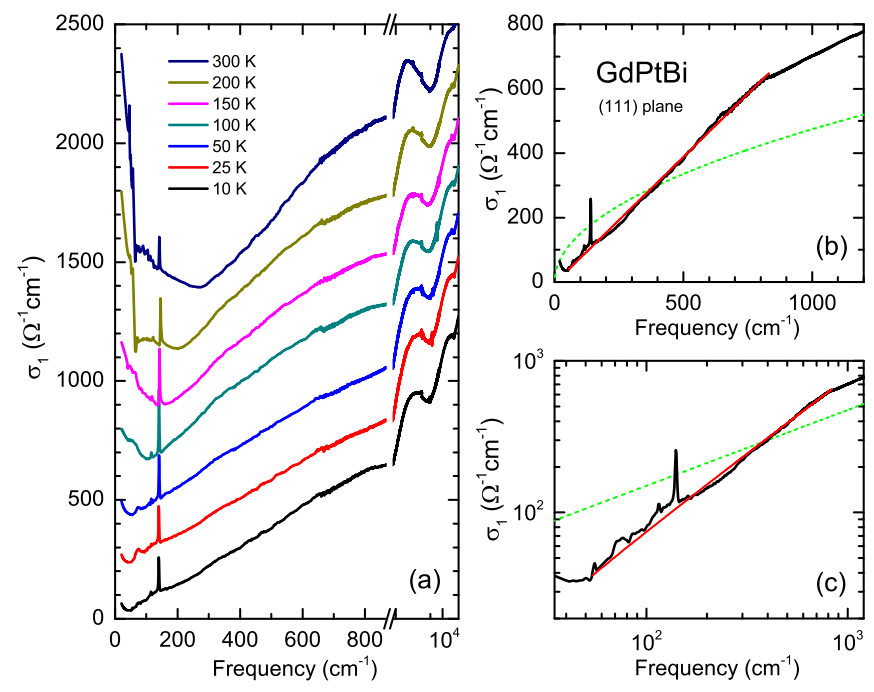

FIG. 3. Optical conductivity of GdPtBi with an emphasis on low frequencies (a). Note a frequency-scale change at 900 $\mathrm{cm}^{-1}$. The curves for $T \geq 25 \mathrm{~K}$ are shifted upwards for clarity (by $200 \Omega^{-1} \mathrm{~cm}^{-1}$ as compered to the previous measurement $T$ ). Linear fit (straight red line) of the experimental $\sigma_{1}(\nu)$ at $10 \mathrm{~K}$ for $50<\nu<800 \mathrm{~cm}^{-1}$ on linear (b) and log$\log$ (c) scales. A square-root behavior of $\sigma_{1}(\nu)$, expected for parabolic bands, is shown by dashed green lines.

$\mathrm{cm}^{-1}, \sigma_{1}(\nu)$ reflects only the interband optical transitions (and the phonons, as mentioned above).

A striking feature of the optical conductivity is its almost perfect linearity in a broad range in the far infrared. This can be seen best in Fig. 3, where experimental conductivity is shown alongside linear fits [square-root behavior of conductivity, expected for parabolic bands, is also shown for comparison]. The behavior of experimental $\sigma_{1}(\nu)$ is basically the same for the three lowest measurement temperatures $(10,25$, and $50 \mathrm{~K})$ : it linearly increases with $\nu$ in the spectral range from approximately 50 up to almost $800 \mathrm{~cm}^{-1}$. The observation of this linearity is an important result of this work. As discussed above, the linearity of the low-energy $\sigma_{1}(\nu)$ is a signature of 3D linear bands [19, 20, 24 27]. However, other band structures may also provide similar $\sigma_{1}(\nu)$. For example, it can be a cumulative effect of many bands with predominantly, but not exclusively, linear dispersion relations. Such a situation was recently reported by some of us for the Weyl semimetal NbP at somewhat higher frequencies [36]. Wavy deviations from a perfectly linear increase of $\sigma_{1}(\nu)$ [see, Figs. 3(b) and 3(c)] indicate that a similar scenario might be realized in GdPtBi.

To get an insight into the origin of linear $\sigma_{1}(\nu)$, we performed band-structure calculations and then computed the interband optical conductivity. In the calculations, we used the linear muffin-tin orbital method (LMTO) 37] as implemented in the relativistic PY LMTO computer code [38, 39]. The Perdew-Burke-Ernzerhof GGA [40] was used for the exchange-correlation potential. The $4 f^{7}$

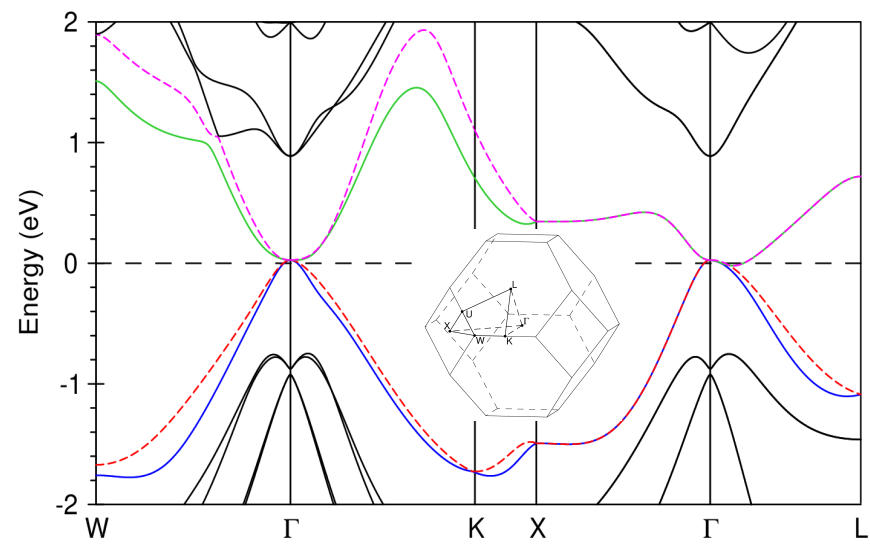

FIG. 4. Band structure of GdPtBi. Low-energy bands are displayed in different colors. The BZ is shown as an inset.

states of gadolinium were treated as semicore states. The $4 f$ spin polarization was not considered in order to model the paramagnetic state studied in this work ( $T \geq 10 \mathrm{~K})$. Relativistic effects, including SOC, were accounted for by solving the four-component Dirac equation inside atomic spheres. BZ integrations were done using the improved tetrahedron method [41].

The calculated band structure is shown in Fig. 4. Our calculations confirm the presence of basically parabolic bands, touching each other at the $\Gamma$ point. A closer look at the low-energy band structure [Figs. 5(a) and 5(b)] reveals the presence of a triple point (marked as $3 \mathrm{p}$ in the figure) along the $\Gamma-L$ line, in agreement with previous calculations [15]. The bands in the plane normal to the $\Gamma-L$ direction possess linear dispersion relations, as shown for the $3 \mathrm{p}-\mathrm{X}$ direction in panel (b). There are eight symmetry-equivalent triple points per BZ. The band structure of GdPtBi near these points is similar to a Weyl or Dirac semimetal with tilted cones, as seen best in panel (a).

Our calculations predict the triple points to be situated $18 \mathrm{meV}$ below the Fermi level. However, the real GdPtBi crystals are known to often possess unintentional doping, which is impossible to control at the crystal-growing stage [12]. Thus, the position of the chemical potential can in reality be within a few tens of $\mathrm{meV}$ off the calculated value. It is instructive to note here that the linear-in-frequency $\sigma_{1}(\nu)$ is expected for tilted cones (of any type), if the chemical potential $\mu$ is situated at the node [42]. In practice, $2 \mu$ ( $\mu$ is measured form the node hereafter) should be below the measurement frequency window. Such a situation can be relevant for our GdPtBi sample, as we discuss below. This is also in agreement with the very small free-carrier (Drude) contribution and low Hall carrier density.

The band structure of GdPtBi in the vicinity of the Fermi level is obviously more complex than the model band structure used in Ref. [42]. Thus, as mentioned 

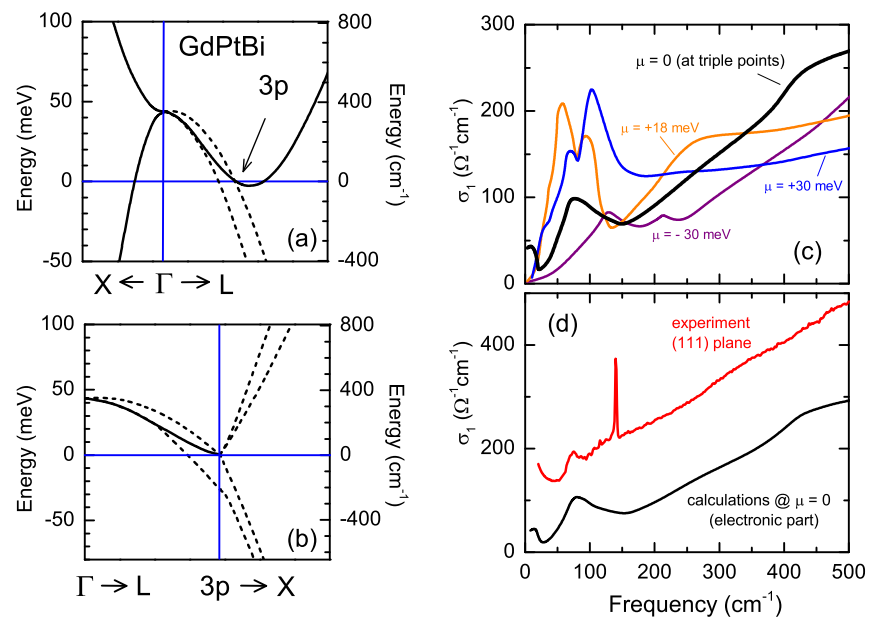

FIG. 5. Panels (a) and (b): Electronic bands of GdPtBi near the triple point (marked as $3 \mathrm{p}$ ). The chemical potential $\mu$ is set to zero at the triple point. Doubly degenerate bands are shown as solid lines, while nondegenerate bands as dashed lines. Panel (c): Calculated interband conductivity of GdPtBi, $\sigma_{1}(\omega)$, for a few different positions of $\mu$ as indicated. Panel (d): Comparison of the measured (upper curve) and calculated for $\mu=0$ (bottom curve) optical conductivity of GdPtBi. Phonon modes are not included in the calculated $\sigma_{1}(\omega)$. The experimental curve is shifted upwards by 100 $\Omega^{-1} \mathrm{~cm}^{-1}$ for clarity.

above, we compute $\sigma_{1}(\nu)$ from the obtained band structure. In these computations, the dipole matrix elements for interband optical transitions were calculated on a $96 \times 96 \times 96 k$-mesh using LMTO wave functions - it is necessary to use sufficiently dense meshes in order to resolve transitions at low energies [43]. The real part of the optical conductivity was calculated using the KuboGreenwood linear-response expressions [44] with the BZ integration performed using the tetrahedron method.

Before we discuss the results of these calculations, we would like to note that obtaining a good agreement between experimental and computed conductivity is known to be challenging [45, 46]. This is particularly the case for (topological) semimetals, where only a qualitative match can typically be achieved $27,36,43,47,48$. In the relevant for this study low-energy part of the spectrum (below $\sim 100 \mathrm{meV}$ ), a reasonable agreement is particularly hard to obtain [27, 43]. Nevertheless, for GdPtBi we have reached a fairly good agreement between our calculations and the experimental spectra at low energies.

The results are shown in Figs. 5(c) and 5(d). Because of the possible carrier doping in GdPtBi, discussed above, we have some freedom in setting the position of the chemical potential. We varied $\mu$ within $\pm 30 \mathrm{meV}$ from the triple point and compared the computed $\sigma_{1}(\nu)$ spectra to each other and to the experiment. Panel (c) demonstrates that the best linear $\sigma_{1}(\nu)$, extrapolating to 0 at $\nu \rightarrow 0$, is obtained, if the chemical potential is at the triple point $(\mu=0)$. If we vary $\mu$, the calculated $\sigma_{1}(\nu)$ either develops huge peaks at low energies $(\nu<200$ $\mathrm{cm}^{-1}$ ), or does not extrapolate to 0 as $\nu \rightarrow 0$, or both. Also, the quasilinear part of the conductivity, calculated for $\mu=0$, spans over the largest frequency range. Thus, we choose the $\mu=0$ curve for further comparison with our experimental results; see panel (d). (Obviously, very small deviations from $\mu=0$ on a meV scale are possible.)

In Fig. 5 (d), a low-temperature (25 K) experimental curve is shown alongside the calculated $\sigma_{1}(\nu, \mu=0)$. The overall linear increase of the experimental curve is well reproduced. It is also evident that both the calculations and experiment provide some deviations from perfect linearity. Most remarkable is the bump, present in the calculations and experiment, at around $80 \mathrm{~cm}^{-1}$. Such deviations reflect the fact that the band structure is not ideally linear in all three directions but more complex. Overall, we can conclude that the observed interband optical conductivity in GdPtBi originates from the transitions between all the bands near the triple points. Linear terms dominate the dispersions of these bands in the close vicinity of the nodes, leading to the almost, but not perfectly, linear optical conductivity in GdPtBi at low frequencies.

From our band-structure calculations, we can compute the Fermi velocities $v_{F}$ for the crossing bands. Calculations exactly at the triple point are technically challenging, and, thus, we compute $v_{F}$ in a close vicinity of it along the $\Gamma-L$ line - at $\pm 0.005 \times 2 \pi / a$ from the triple point; here $a$ is the lattice constant. For the doubly degenerate electronlike band, we obtain $v_{F}=1.1$ and $0.5 \times 10^{5} \mathrm{~m} / \mathrm{s}$, while for the nondegenerate holelike band $v_{F}=2.4$ and $2.8 \times 10^{5} \mathrm{~m} / \mathrm{s}$.

In a simple model of electron-hole symmetric crossing linear electronic bands, the optical conductivity is related to the Fermi velocity $v_{F}$ via [19, 20] $\sigma_{1}(\omega)=\frac{e^{2} g N}{24 h} \frac{\omega}{v_{F}}$, where $g$ is the band degeneracy at the crossing point (e.g., a Dirac node has $g=4$ ) and $N$ is the number of nodes per BZ. Obviously, this simple formula has a very limited applicability. Nevertheless, if we straightforwardly apply it to our experimental $\sigma_{1}(\omega)$ and set $g=3$ and $N=8$, we obtain an averaged Fermi velocity of $\sim 10^{5} \mathrm{~m} / \mathrm{s}$, which is in good agreement with the values calculated above.

In summary, we have found the low-frequency optical conductivity of GdPtBi to be linear in a broad frequency range $\left(50-800 \mathrm{~cm}^{-1}, \sim 6-100 \mathrm{meV}\right.$ at $\left.T \leq 50 \mathrm{~K}\right)$. This linearity strongly suggests the presence of threedimensional linear electronic bands with band crossings near the chemical potential. A comparison of our data with the optical conductivity computed from the band structure demonstrates that the observed $\sigma_{1}(\omega)$ originates from the transitions near the triple points. From the optical spectra, we directly determine the plasma frequency of free carriers in GdPtBi and estimate an averaged Fermi velocity at the nodes: $v_{F} \sim 10^{5} \mathrm{~m} / \mathrm{s}$. The values of $v_{F}$, calculated from the band structure near the triple points along the $\Gamma-L$ line, range from 0.5 to 
$2.8 \times 10^{5} \mathrm{~m} / \mathrm{s}$ depending on the band and the momentum direction.

We are grateful to Ece Uykur and Dominik Günther for valuable experimental support, to Gabriele Untereiner for technical assistance, and to Johannes Gooth for many fruitful discussions. This work was funded by DFG via grant No. DR228/51-1.

[1] L. Wollmann, A. K. Nayak, S. S. P. Parkin, and C. Felser, Annu. Rev. Mater. Res. 47, 247 (2017).

[2] K. Manna, Y. Sun, L. Müchler, J. Kübler, and C. Felser, Nat. Rev. Mater. 3244 (2018).

[3] F. Casper, T. Graf, S. Chadov, B. Balke, and C. Felser, Semicond. Sci. Technol. 27, 063001 (2012).

[4] S. Chadov, X. Qi, J. Kübler, G. H. Fecher, C. Felser, and S. C. Zhang, Nat. Mater. 9, 541 (2010).

[5] H. Lin, A. Wray, Y. Xia, S. Xu, S. Jia, R. J. Cava, A. Bansil, and M. Z. Hasan, Nat. Mater. 9, 546 (2010).

[6] D. Xiao, Y. Yao, W. Feng, J. Wen, W. Zhu, X.-Q. Chen, G. M. Stocks, and Z. Zhang, Phys. Rev. Lett. 105, 096404 (2010).

[7] W. Al-Sawai, H. Lin, R. S. Markiewicz, L. A. Wray, Y. Xia, S.-Y. Xu, M. Z. Hasan, and A. Bansil, Phys. Rev. B 82, 125208 (2010).

[8] C. Liu, Y. Lee, T. Kondo, E. D. Mun, M. Caudle, B. N. Harmon, S. L. Bud'ko, P. C. Canfield, and A. Kaminski, Phys. Rev. B 83, 205133 (2011).

[9] Z. K. Liu, L. X. Yang, S.-C. Wu, C. Shekhar, J. Jiang, H. F. Yang, Y. Zhang, S.-K. Mo, Z. Hussain, B. Yan, C. Felser, and Y. L. Chen, Nat. Commun. 7, 12924 (2016).

[10] J. A. Logan, S. J. Patel, S. D. Harrington, C. M. Polley, B. D. Schultz, T. Balasubramanian, A. Janotti, A. Mikkelsen, and C. J. Palmstrøm, Nat. Commun. 7, 11993 (2016).

[11] C. Shekhar, A. K. Nayak, S. Singh, N. Kumar, S.C. Wu, Y. Zhang, A. C. Komarek, E. Kampert, Y. Skourski, J. Wosnitza, W. Schnelle, A. McCollam, U. Zeitler, J. Kübler, S. S. P. Parkin, B. Yan, and C. Felser, arXiv:1604.01641.

[12] M. Hirschberger, S. Kushwaha, Z. Wang, Q. Gibson, S. Liang, C. A. Belvin, B. A. Bernevig, R. J. Cava, and N. P. Ong, Nat. Mater. 151161 (2016).

[13] S. Liang, J. Lin, S. Kushwaha, J. Xing, N. Ni, R. J. Cava, and N. P. Ong, Phys. Rev. X 8, 031002 (2018).

[14] N. Kumar, S. N. Guin, C. Felser, and C. Shekhar, Phys. Rev. B 98, 041103 (2018).

[15] H. Yang, J. Yu, S. S. P. Parkin, C. Felser, C.-X. Liu, and B. Yan, Phys. Rev. Lett. 119, 136401 (2017).

[16] Z. Zhu, G. W. Winkler, Q. S. Wu, J. Li, and A. A. Soluyanov, Phys. Rev. X 6, 031003 (2016).

[17] M. Dressel and G. Grüner, Electrodynamics of Solids (Cambridge University Press, Cambridge, 2002).

[18] T. Ando, Y. Zheng, and H. Suzuura, J. Phys. Soc. Jpn. 71, 1318 (2002).

[19] P. Hosur, S. A. Parameswaran, and A. Vishwanath, Phys. Rev. Lett. 108, 046602 (2012).

[20] A. Bácsi and A. Virosztek, Phys. Rev. B 87, 125425 (2013).

[21] J. P. Carbotte, J. Phys. Condens. Matter 29, 045301
(2017)

[22] S. P. Mukherjee and J. P. Carbotte, Phys. Rev. B 95, 214203 (2017).

[23] S. Ahn, E. J. Mele, and H. Min, Phys. Rev. Lett. 119, 147402 (2017).

[24] R. Y. Chen, S. J. Zhang, J. A. Schneeloch, C. Zhang, Q. Li, G. D. Gu, and N. L. Wang, Phys. Rev. B 92, 075107 (2015).

[25] B. Xu, Y. M. Dai, L. X. Zhao, K. Wang, R. Yang, W. Zhang, J. Y. Liu, H. Xiao, G. F. Chen, A. J. Taylor, D. A. Yarotski, R. P. Prasankumar, and X. G. Qiu, Phys. Rev. B 93, 121110 (2016).

[26] D. Neubauer, J. P. Carbotte, A. A. Nateprov, A. Löhle, M. Dressel, and A. V. Pronin, Phys. Rev. B 93, 121202 (2016).

[27] S. I. Kimura, H. Yokoyama, H. Watanabe, J. Sichelschmidt, V. Süß, M. Schmidt, and C. Felser, Phys. Rev. B 96, 075119 (2017).

[28] Y. Shao, Z. Sun, Y. Wang, C. Xu, R. Sankar, A. J. Breindel, C. Cao, M. M. Fogler, F. Chou, Z. Li, T. Timusk, M. B. Maple, and D. N. Basov, arXiv:1806.01996.

[29] P. C. Canfield, J. D. Thompson, W. P. Beyermann, A. Lacerda, M. F. Hundley, E. Peterson, and Z. Fisk, J. Appl. Phys. 70, 5800 (1991).

[30] C. C. Homes, M. Reedyk, D. A. Cradles, and T. Timusk, Appl. Opt. 32, 2976 (1993).

[31] D. B. Tanner, Phys. Rev. B 91, 035123 (2015)

[32] M. B. Schilling, A. Löhle, D. Neubauer, C. Shekhar, C. Felser, M. Dressel, and A. V. Pronin, Phys. Rev. B 95, 155201 (2017).

[33] M. B. Schilling, L. M. Schoop, B. V. Lotsch, M. Dressel, and A. V. Pronin, Phys. Rev. Lett. 119, 187401 (2017).

[34] Similar fitting procedures can, in principle, be utilized even as substitutes of the Kramers-Kronig analysis; see A. B. Kuzmenko, Rev. Sci. Instrum. 76, 083108 (2005) and G. Chanda, R. P. S. M. Lobo, E. Schachinger, J. Wosnitza, M. Naito, and A. V. Pronin, Phys. Rev. B 90, 024503 (2014).

[35] Z. V. Popović, G. Kliche, R. Liu, and F. G. Aliev, Solid State Commun. 74, 829 (1990); P. Hermet, K. Niedziolka, and P. Jund, RSC Adv. 3, 22176 (2013); C. Çoban, K. Çolakoğlu, and Y. Ö. Çiftçi, Phys. Scr. 90, 095701 (2015); D. Shrivastava and S. P. Sanyal, Physica C 544, $22(2018)$.

[36] D. Neubauer, A. Yaresko, W. Li, A. Löhle, R. Hübner, M. B. Schilling, C. Shekhar, C. Felser, M. Dressel, and A. V. Pronin, arXiv:1803.09708.

[37] O. K. Andersen, Phys. Rev. B 12, 3060 (1975).

[38] V. N. Antonov, A. Y. Perlov, A. P. Shpak, and A. N. Yaresko, J. Magn. Magn. Mater. 146, 205 (1995).

[39] V. Antonov, B. Harmon, and A. Yaresko, Electronic structure and magneto-optical properties of solids (Kluwer Academic, Dordrecht, 2004).

[40] J. P. Perdew, K. Burke and M. Ernzerhof, Phys. Rev. Lett. 77, 3865 (1996).

[41] P. E. Blöchl, O. Jepsen, and O. K. Andersen, Phys. Rev. B 49, 16223 (1994).

[42] J. P. Carbotte, Phys. Rev. B 94, 165111 (2016).

[43] D. Chaudhuri, B. Cheng, A. Yaresko, Q. D. Gibson, R. J. Cava, and N. P. Armitage, Phys. Rev. B 96, 075151 (2017).

[44] C. S. Wang and J. Callaway, Phys. Rev. B 9, 4897 (1974).

[45] P. Y. Yu and M. Cardona, Fundamentals of Semiconduc- 
tors: Physics and Materials Properties (Springer, Berlin, 2010).

[46] D. Santos-Cottin, Y. Klein, Ph. Werner, T. Miyake, L. de' Medici, A. Gauzzi, R. P. S. M. Lobo, and M. Casula, Phys. Rev. Mater. 2, 105001 (2018).

[47] D. Grassano, O. Pulci, A. M. Conte, and F. Bechstedt,
Sci. Rep. 8, 3534 (2018).

[48] A. J. Frenzel, C. C. Homes, Q. D. Gibson, Y. M. Shao, K. W. Post, A. Charnukha, R. J. Cava, and D. N. Basov, Phys. Rev. B 95, 245140 (2017). 